\title{
Optimization of Energy Consumption in Clustered Nodes of WSN Using the Black Hole Algorithm
}

\author{
Masoum Farahmandian ${ }^{1}$ and Abdolreza Hatamlou ${ }^{2}$ \\ ${ }^{1}$ Department of Computer Engineering, Urmia Branch, Islamic Azad University, Urmia, Iran \\ ${ }^{2}$ Department of Computer Engineering, Khoy Branch, Islamic Azad University, Khoy, Iran \\ E-mail: 'gmasafat@gmail.com, hatamlou@iaukhoy.ac.ir
}

\begin{abstract}
Nowadays due to the low cost and easy communication, Wireless Sensor Networks (WSN) have many uses for supervisory activities in a variety of environments. The limitation of the energy available in the nodes of the networks is the main problem that affects the survival of the network. Studies have shown that with the creation of control on the number and location of the cluster heads on, the efficiency of energy can increase which results in increase of the life of the network. However, due to the dynamic nature of the issue and due to continuous changes in cluster heads in any of the network activity, this issue is not applicable to classical methods of modeling. In this study using black hole algorithm (BHA), we determine the number and location of the cluster heads to the best optimization. Fitting the criterion will be based on the minimum consumed energy of each node in the network in every data transmission operation period that will be led to the creation of a balance in energy consumption of cluster heads and consequently longer life of network.
\end{abstract}

Keywords: Wireless Sensor Networks, BHA, Classification of Clusters, Cluster Heads, Energy Balance, Optimization.

\section{INTRODUCTION}

In recent years, the electronic and telecommunication technologies development, has led to the construction of the small and relatively inexpensive sensors. In spite of their small size, these sensors have the ability of calculation, data measurement and programming which are in connection with each other by a wireless network $[1,2]$. These networks called WSN are used to extract data from environment and monitoring of the events. The applications of these networks in different fields are increasing daily [3]. The fundamental challenge in the design of the network is the energy source limitation of sensors that affect the survival of network. In addition, due to very high number of sensors in the network and the possibility of lack of access to them, replacement or recharging sensors' batteries is not practical. For this reason, providing procedures which are able to reduce the communicational nodes intervals for cutting down the energy consumption and increase in the life of the network, is very vital [4].
Studies have shown that the organization of the network nodes in clusters brings higher efficiency of energy which results in the increase of the network life. The period of time until the death of the first or last node of the network is called the network's lifetime [5]. One of the proposed protocols in sensor networks in cluster classification is called LEACH Protocol that has been formed of two establishing and permanent phases [6]. In permanent mode phase, the data transmission will be in the form of single-step form. In each cluster some nodes are selected as the cluster heads. The collected data from the nodes of members is processed in head cluster locally before posting to the base station, and additional underlying data- if any exists- will be deleted, and then in the form of a new package will be sent to the base station.

Figure 1 shows a sample of a wireless sensor network with sending data from nodes to head clusters and also from head clusters to the base station and vice versa. 


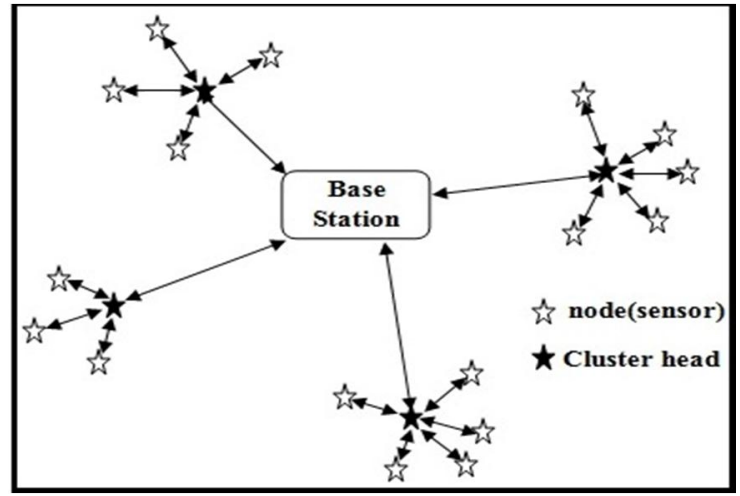

Fig. 1. Sending data from the head clusters to base station in wireless sensor network

Since the consumption energy of clusters due to exchange of many data is more than normal nodes, it is clear that their energy should be finished in a shorter time too. Therefore, in LEACH dynamic clustering has been proposed, in order to, solve the problem of rapid finishing of cluster heads' energy. This means that after each start-up phase i.e. after each period of operation of sending and receiving data, head clusters change and another node randomly is selected as cluster head. However, the problem that occurs in this mode (random selection of head clusters) is the inappropriate distribution of head clusters in the network; this means that in part of the covered zone there may be two or more cluster heads while there is no cluster head in another zone (Figure 2).

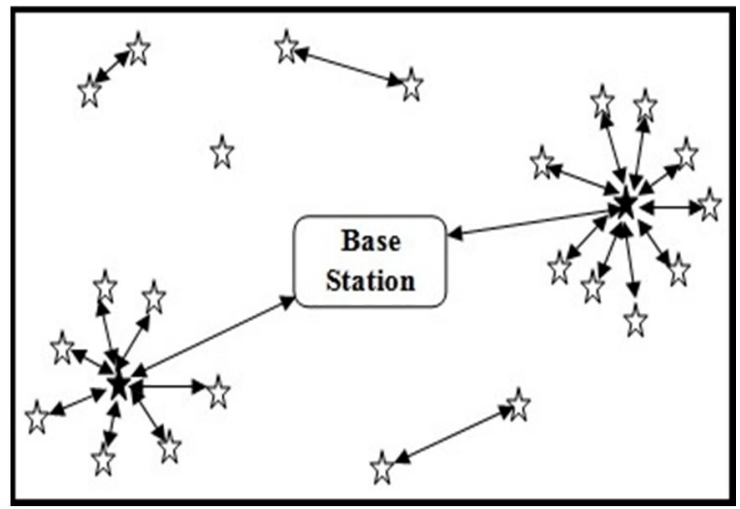

Fig. 2. Random Selection of Head Clusters in LEACH

\subsection{Problem}

Establishing control over the number and location of cluster heads and also clusters size according to the number of members has always been a big challenge. Solving this problem needs efficient and effective clustering algorithms to reduce the energy consumption and balancing the network load [7].
Dynamic nature of problem because of continuous changes in cluster heads in each period of network activity makes the problem more complex which will not be modeling with the classical methods of mathematics. The number and location of clusters that have the best efficiency in energy consumption in the network is an NP_hard problem. The common cluster classification algorithms in other articles usually have scientific methods which the Global reply is not seen in none of them. On the other hand, BHA in solving dynamic problems is very flexible. In this study, using the BHA, we determine the location of head clusters so that the energy consumption reduces to the minimum in the network. Fitting the criterion will be based on the minimum consumed energy from nodes in the network in every generation.

\section{SYSTEM AND MODEL ENERGY}

Considered networks have the following characteristics:

- All of sensor nodes are the same and are distributed in whole network and whole square area regularly.

- The base situation is settled in a location out of the square area and in a far distance. Selection of the station location depends on the basis of the application [8].

- Telecommunication channel is assumed symmetrical and multi-path model.

- The nodes are able to regulate their transition power nodes according to their distance to the desired recipient. This is essential to ensure the network integrity [9].

- The nodes all have equal energy and ability.

- Location and identification of all nodes are clear for the base station.

Hainzlman provided a model for energy consumption as follows [10]. Each node for sending $l$ bit to a $d$ distance, consumes $E s$ the energy that comes from the following relation:

$E_{s}$
$= \begin{cases}l E_{\text {elect }}+l \varepsilon_{f s} d^{2} & d \geq d_{c o} \\ l E_{\text {elect }}+l \varepsilon_{\text {mp }} d^{4} & d \geq d_{c o}\end{cases}$

Where Eelect is the necessary energy for activation of sender's electronic circuits. dco is a threshold. $\varepsilon f s$ and $\varepsilon m p$ are the activation power amplifier of energy for two situation of multi-path 
and open space. In case of being more distance from the threshold $d c o$, sender uses the multi-path model with the regulation of power amplifier; otherwise the open space model is used for the channel.

Also, the amount of energy to get this, $l$ bit is spent in the client node:

$$
E_{r}=l E_{\text {elect }}
$$

\section{THE PROPOSED METHOD}

\subsection{Startup phase}

The use of centralized algorithms in wireless sensor networks' applications helps to use intelligent and non-intelligent optimization algorithms and change their parameters during the performance. In this article, the assumption is that all operations of the establishment and management of clusters are done in the base station. Base station as a powerful processor with unlimited energy center is capable to classify the received data and location of network nodes in clusters in balance with whole energy consumption energy. The aim is to distribute the created structure resulted from nodes and some head clusters in a way that the total energy consumption of network is minimized.

\subsection{Steady State Phase}

After the formation of clusters, they recognize the nodes in other head clusters and send their own data according to the scheduling program TDMA (Time Division Multiple Access), which head clusters, determine and send them. Each time the implementation phase of the startup and steady state is called "round". The cluster is done again and new nodes are selected for the role of head clusters at the end of each round [11].

\subsection{The Details of the Algorithm}

As mentioned, it is assumed that all cluster classification operations are conducted within the basic station then the result is sent to the entire network. In base station the network designer can determine the number of required head clusters; this number determines the number of the stars. Each of the stars is the identification of some network nodes that their energy is more than the network energy. We should bear in mind that the base station is aware of each nodes' energy; therefore, they can calculate the average of network energy.
The structure of the stars in this case is defining as follows:

$$
\text { Stars }=\left\{g_{i} \mid i=1,2, \ldots L\right\}
$$

Where $\mathrm{L}$ is the number of the stars and $g_{i}$ is the $\mathrm{i}^{\mathrm{ed}}$ star. In this article the real and continuous coding is used for each star from. Therefore, the space of the stars will be defined as follows:

$\mathrm{g}_{\mathrm{i}}=\mathrm{S} . \mathrm{ID} \quad \mathrm{ID}=1,2, \ldots, \mathrm{N}$

Where: S.residual_Energy $\geq \mathrm{E}_{\text {mean }}$

Where S.ID and S.residual_Energy are expressive of the remaining energy and identification of node. $\mathrm{N}$ is the total number of nodes and $\mathrm{E}_{\text {mean }}$ is the average of network energy. After the implementation of the proposed BHA [12], the base station that makes the least amount of fitness function (or in other words, the least network energy differences in comparison with the previous round) and its current nodes are introduced as head cluster (black hole) to the network. If we present the current energy network at $\mathrm{K}^{\text {th }}$ round with $E_{\mathrm{Network}}^{\mathrm{k}}$, the fitness function will have the following values which should be minimized:

$$
\text { fitness }=\left|E_{\text {Network }}^{\mathrm{k}}-\mathrm{E}_{\mathrm{Network}}^{\mathrm{k}-1}\right|
$$

In this respect, the mark || shows the absolute value. Other steps of algorithm such as the creation of the cluster, scheduling program and sending have been assumed in accordance with the LEACH algorithm.

\section{SIMULATION AND RESULT}

We have stimulated the proposed algorithm and LEACH Protocol using MATLAB simulation software. The given values considered in this simulation are shown in table 1 .

Table 1: Simulation Parameters

\begin{tabular}{cc}
\hline Network Size & $100 \mathrm{~m}$ \\
\hline Number Nodes(N) & 100 \\
\hline Primary Energy & $0.1 \mathrm{~J}$ \\
\hline $\mathrm{E}_{\text {elect }}$ & $50 \mathrm{~nJ} / \mathrm{bit}$ \\
\hline$\varepsilon_{\mathrm{fs}}$ & $10 \mathrm{pJ} / \mathrm{bit} / \mathrm{m}^{2}$ \\
\hline $\mathrm{D}_{\text {crossover }}=\mathrm{d}_{\mathrm{co}}$ & $87 \mathrm{~m}$ \\
\hline $\mathrm{E}_{\mathrm{DA}}$ & $5 \mathrm{~nJ} / \mathrm{bit} / \mathrm{signal}$ \\
\hline Packets size & $4000 \mathrm{bits}$ \\
\hline
\end{tabular}

Table 2 shows the used parameters in BHA. 
Table 2: The Values of BHA

\begin{tabular}{cc}
\hline Primary Population & 100 \\
\hline Number of iterations & 100 \\
\hline
\end{tabular}

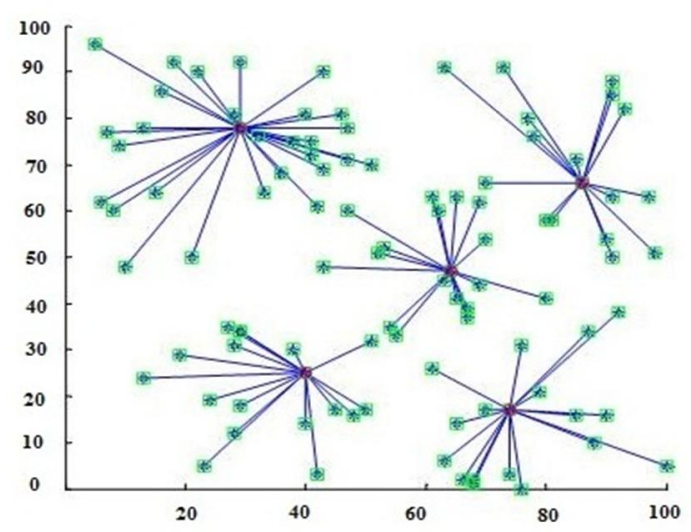

Fig. 3. An example of algorithm responses with formed clusters

Figure 3 shows an example of the algorithm's responses. As we see, the head clusters distribution is better than LEACH and head clusters have been distributed steady throughout the network. Whereas, in LEACH, there is the possibility of head clusters accumulation or the emptiness of a region from head cluster.

In Figure 4, the proposed algorithm and LEACH are compared in terms of the rate of network energy reduction during the 150 rounds sending and receiving data. As it is seen, our algorithm has made the rate of energy reduction in network steadier and more consistent than LEACH. Unsteady reduction of network energy in LEACH and imbalance in energy consumption of head clusters are resulted in a reduction in useful lifetime of network.

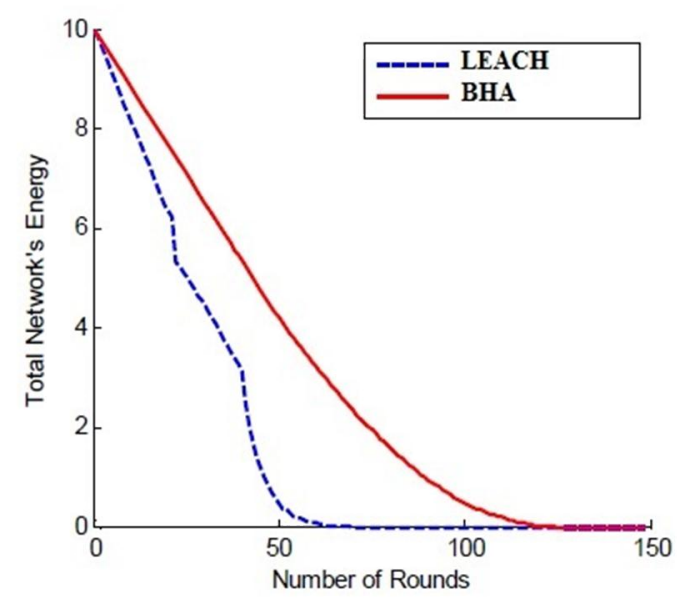

Fig. 4. The rate of energy reduction in network during its life.
Improvement of the algorithm performance in terms of increasing of the longevity of the sensor network compared to LEACH is specified in Figure 5. As it is shown, the proposed algorithm increases the death time of the first node significantly in comparison with the LEACH. Even if after the death of the first node in the network, the network is still given the permission to continue working until all the nodes of the network lose their energy, we will see that after the death of the last node, the number of rounds is still further.

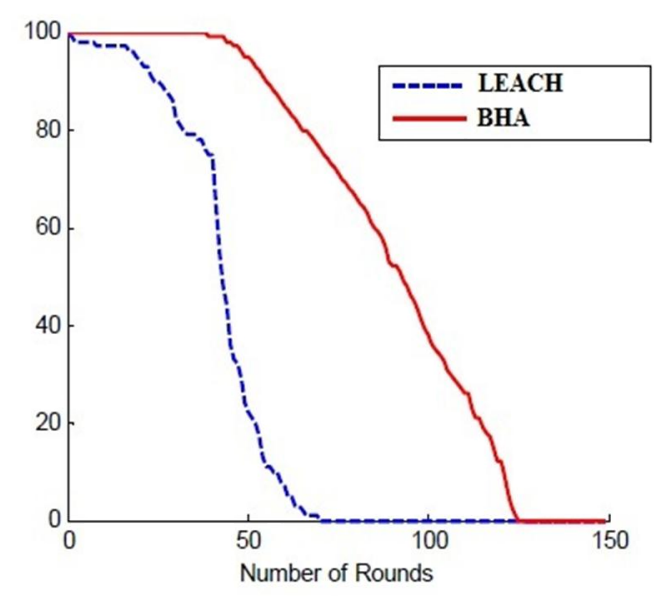

Fig. 5. Compare the number of live sensors in network.

It is specified from both Figures 4 and 5, using $\mathrm{BH}$ in the selection of head clusters algorithm given the minimum energy difference in comparison with the previous step as the fitness function, consumes the present energy in nodes steadily; this cause an increase in sensor network's lifetime.

\section{CONCLUSION}

Optimum energy consumption in WSN is important, in a way that the optimum energy consumption increases the life of the network. In this study, using BH algorithm which is performed centrally in base station, determine the location of head clusters in order to have the minimum energy consumption. We have considered the fitting criteria based on minimum consumed energy of network's nodes during each sending data operation. Development of balance and steadiness in the energy consumption of the nodes and longer life of network is the achievement of using $\mathrm{BH}$ algorithm in this study. 


\section{REFERENCES}

[1] Heinzelman, W. B., A. P. Chandrakasan, et al. (2002). "An application-specific protocol architecture for wireless microsensor networks." Wireless Communications, IEEE Transactions on 1(4): 660-670.

[2] Tilak, S., N. B. Abu-Ghazaleh, et al. (2002). "A taxonomy of wireless micro-sensor network models." ACM SIGMOBILE Mobile Computing and Communications Review 6(2): 28-36.

[3] Ren, K., W. Lou, et al. (2007). "On broadcast authentication in wireless sensor networks." Wireless Communications, IEEE Transactions on 6(11): 4136-4144.

[4] Zhang, Y., L. T. Yang, et al. (2010). RFID and sensor networks: architectures, protocols, security, and integrations, CRC Press.

[5] Younis, O., M. Krunz, et al. (2006). "Node clustering in wireless sensor networks: recent developments and deployment challenges." Network, IEEE 20(3): 20-25.

[6] Katiyar, V., N. Chand, et al. (2011). Improvement in LEACH protocol for largescale wireless sensor networks. Emerging Trends in Electrical and Computer Technology (ICETECT), 2011 International Conference on, IEEE.

[7] Chen, H., H. Mineno, et al. (2006). A metadata-based data aggregation scheme in clustering wireless sensor networks. Mobile Data Management, 2006. MDM 2006. 7th International Conference on, IEEE.

[8] Hou, Y. T., Y. Shi, et al. (2006). "Optimal base station selection for anycast routing in wireless sensor networks." Vehicular Technology, IEEE Transactions on 55(3): 813-821.

[9] Jia, W. and J. Wang (2006). "Analysis of connectivity for sensor networks using geometrical probability." IEE ProceedingsCommunications 153(2): 305-312.

[10]Heinzelman, W. B. (2000). Applicationspecific protocol architectures for wireless networks, Massachusetts Institute of Technology.

[11] Mohamed-Lamine, M. (2013). New clustering scheme for wireless sensor networks. Systems, Signal Processing and their Applications (WoSSPA), 2013 8th International Workshop on, IEEE.

[12] Hatamlou, A. (2012). "Black hole: A new heuristic optimization approach for data clustering." Information sciences: 175-184. 